\title{
STATE OF THE ART MOLECULAR BEAM EPITAXY OF III-V COMPOUNDS
}

\author{
C.T. Foxon \\ Department of Physics, University of Nottingham, Nottingham NG7 2RD, England \\ and also Department of Physics, Technical University of Eindhoven, The Netherlands
}

This paper discusses molecular beam epitaxy with particular emphasis on the production of state of the art electronic and optoelectronic low dimensional structures and devices. The molecular beam epitaxy process is outlined briefly and the practical problems associated with producing "state of the art" (Al,Ga)As/GaAs structures are considered. Examples include high mobility electron and hole gases, low threshold current lasers and the multi-quantum well solar cells.

PACS numbers: $61.50 . C j, 68.55 . B d, 72.20 .-\mathrm{i}, 85.60 .-\mathrm{q}$

\section{Introduction}

Molecular beam epitaxy (MBE) is a refined form of evaporation in which molecular beams interact on a heated substrate under ultra-high vacuum conditions to produce an epitaxial film. Growth rates in MBE are typically 1 to 2 monolayers per second which corresponds to a pressure at the substrate of about $10^{-6}$ millibars. For even moderately pure films to be produced it is necessary for the partial pressure of the unwanted species which might stick to the film to be in the $10^{-12}$ millibar pressure regime, hence the need for vacuum quality.

\section{Procedures for obtaining high quality (Al,Ga)As/GaAs films}

The first requirement for good quality material is the correct preparation of the MBE equipment. In practice this means first outgassing the "Knudsen cells" at high temperature to remove impurities and then selecting the best chemicals for evaporation. Here the main difficulty is that the manufacturers quote only impurity levels for metallic elements, but do not estimate the unwanted carbon or oxygen content, which is of vital importance. As a result the MBE community has learned over the years, from experiences world-wide, which materials to use and which to avoid.

The next important point is to prepare the system to remove trace water vapour and other oxides before growth, here in situ monitoring with a mass spectrometer during bake-out is helpful especially in finding small leaks, especially those which open during the heating or cooling process. 
All of this rather dull, routine, but essential work distinguishes those laboratories able to produce high quality samples from those unable to do so [1].

Introducing and removing samples whilst maintaining the integrity of the vacuum is another important factor in determining sample quality. It is essential to rcmove water vapour from both the substrate and its holder by heating to $400^{\circ} \mathrm{C}$ for an extended period in the preparation chamber, but it is equally important to allow the vacuum to recover before opening the valve to the growth chamber [1]. Choice of optimum growth rates, group V:III hux ratio and substrate temperature are also important in some situations.

\section{High mobility two-dimensional electron and hole gases}

A two-dimensional electron (hole) gas (2DEG/2DIIG) is formed close to the hetero-interface of a narrow band gap material grown before or after $n(p)$-doped wider band gap material. Electrons (holes) are effectively transferred from the wide band gap material until the Fermi levels are equalised giving rise to band bending in both the wider and narrow band gap materials, thus separating the electrons (holes) from the dopant atoms. Further enhancement can be brought about by introducing an undoped spacer layer (sometimes referred to as a "set-back") between the doped region and the interface. The electrons (holes) are free to move in the plane perpendicular to the growth direction, but confined to a narrow region ( 5 to $10 \mathrm{~nm}$ ) close to the hetero-interface. At low temperatures, when the phonon scattering rate is reduced, very high mobility 2DEG (2DIIG) structures can be obtained and in samples of this type, studies of new states of matter such as the fractional quantum IIall effect and Wigner solid have been undertaken in recent years. Because, at the surfaces of $\mathrm{GaAs}$ and $(\mathrm{AlGa})$ As the Fermi level is pinned mid-gap, additional dopant atoms must be added to the wider band gap material to achieve an electrically active structure, but if the doped region is too thick, free electrons in the (AIGa)As will give rise to a parallel conducting path. This is not a problem for 2DIIG structures due to freeze out of carriers holes resulting from the depth of the acceptors.

Most optimum high mobility structures have been obtained in ( $\mathrm{AlGa}) \mathrm{As}$ / GaAs samples at the "normal" interface, where the wide band gap (AlGa)As is grown after the undoped GaAs. The inferior properties of the "inverted" interface structures, grown in the opposite order, can be traced mainly to segregation of dopant atoms from the wide band gap ( $\mathrm{AlGa}$ )As into the 2DEG/2DIIG region close to the hetero-interface, this point will be considered below briefly.

For 2DEGs the key to improved performance comes from considering the various factors which can degrade the mobility and they include scattering by local residual impurities in the GaAs, scattering by both dopant atoms and unintentional impurities in the wider band (AlGa)As and by the roughness of the hetero-interface.

\subsection{IIigh mobility GaAs}

Undoped (or strictly unintentionally doped) films grown by MBE using $\mathrm{As}_{4}$ are generally lightly $p$-type, but very closely compensated. Using the smallest amount of $\mathrm{Si}$ as an $n$-type dopant gives material with electron mobilities at $77 \mathrm{~K}$ in 
excess of $10^{5} \mathrm{~cm}^{2} \mathrm{~V}^{-1} \mathrm{~s}^{-1}$ if the appropriate procedures discussed above are used. The limiting factor is usually residual carbon (identified using low temperature photoluminescence studies) and the amount can be reduced in a number of ways. Simply growing thick films of GaAs enabled high quality 2DEGs to be obtained routinely [2] and later we observed, in common with others, that GaAs with a very low carbon content could be obtained using by introducing superlattices (SLs) before the active part of the structure [3]. This suggests that a SL traps carbon which would otherwise segregate during growth and SIMS studies confirm that this occurs at the "inverted interface". SLs are now used in all high mobility 2DEG/2DHG samples.

The use of $\mathrm{As}_{2}$ has also resulted in improved mobility in unintentionally doped films, which are usually $n$-type using the dimer, resulting in peak mobilities greater than $4.5 \times 10^{5} \mathrm{~cm}^{2} \mathrm{~V}^{-1} \mathrm{~s}^{-1}[4]$.

\subsection{High mobility 2DEGs}

In addition to reducing the scattering due to residual impurities in the GaAs, attention to the quality of the undoped and intentionally doped (AlGa)As regions is essential: Several schemes have been used to minimise the scattering due to dopant atoms, we have employed lightly doped (AlGa)As to move the ionised impurities further from the 2DEG and hence increase the mobility at low tem-

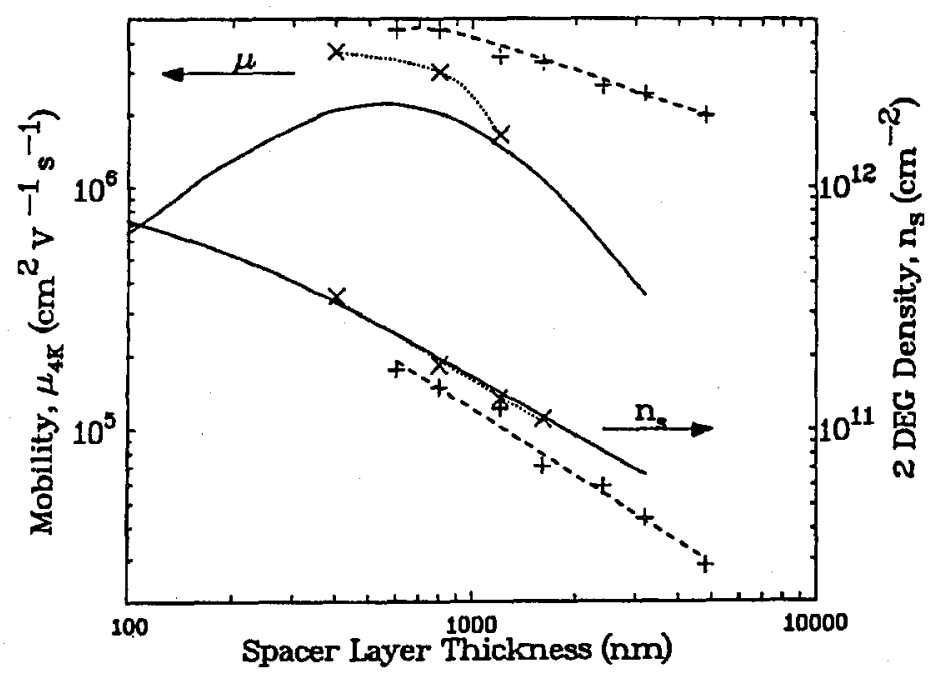

Fig. 1. The effects of the undoped spacer layer thickness on the two-dimensional electron gas density and mobility (at $4 \mathrm{~K}$ ) for a variety of structures. The beneficial effects on introducing a superlattice into the GaAs buffer layer and of replacing the heavily doped $(\mathrm{AlGa})$ As with a thicker more lightly doped layer can clearly be seen by comparision with the solid lines which show data for similar samples with a heavy doped (AlGa)As layer and no superlattice. 
perature $(<1 \mathrm{~K})[5]$. Similar results have been obtained using a double $\Delta$-doped arrangement with a low density close to the 2DEG and a high density nearer the surface $[6,7]$. The improvements resulting from the use of SLs in the GaAs and lower doping in the (AlGa)As are illustrated in Fig. 1. For a narrow spacer layer sample, the scattering rate is determined by the remote impurities in the intentionally doped (AlGa)As, but for wider spacer layer structures the quality of the $\mathrm{GaAs}$ and the interface roughness are key factors. A peak in the mobility curve occurs at about $60 \mathrm{~nm}$ where the limitations due to the quality of the GaAs and $(\mathrm{AlGa})$ As are roughly equivalent.

Choice of growth temperature also plays a role in the optimization process, the active part of the structure needs to be grown at about $630^{\circ} \mathrm{C}$ which appears to minimise unwanted impurities and give a good hetero-interface. Some workers, particularly using the $\Delta$-doped approach have then reduced the temperature for this region to minimise the diffusion and segregation in this part of the structure.

In all such structures the density of electron can be increased by illumination due to the persistent photoconductivity observed in such structures, associated in part with the DX centres in the (AlGa)As. In all high quality 2DEGs, this results in an increase in low temperature mobility partly due to the increased Fermi velocity and also due to increased screening. Such studies indicate that even in the very best structure scattering by remote and local ionised impurities is the dominant process limiting performance. At present this restricts the mobility to about $10^{7} \mathrm{~cm}^{2} \mathrm{~V}^{-1} \mathrm{~s}^{-1}$ [5-7] at milliKelvin temperatures.

\subsection{High mobility 2DHGs}

All of the above structures were grown on (001) oriented substrates and attempts to obtain equivalent performance for 2DHGs using Be as the dopant were disappointing, even taking into account the increased hole mass. Some improvement was obtained by reducing the Be doping level, but the real breakthrough came with the growth on (311)A surfaces using $\mathrm{Si}$ as the dopant $[8,9]$, which had been previously shown to result in $p$-type films due to the different bonding sites present on this surface [10].

Using some sources of arsenic, samples exhibit persistent photoconductivity effects similar to those observed in 2DEGs, but not anticipated for the 2DHG due to the absence of DX centres in $p$-type (AlGa)As. The mobility in such 2DHGs is largely independent of hole density in marked contrast to the 2DEG behaviour. This suggests that the dominant scattering mechanism in the 2DHG may not be due to ionised impurities, but to interface roughness. At present the best mobilities for 2DHGs is about $10^{6} \mathrm{~cm}^{2} \mathrm{~V}^{-1} \mathrm{~s}^{-1}[9]$ at milliKelvin temperatures and here there is a much stronger temperature dependence below $4 \mathrm{~K}$ than in comparable quality 2DEGs.

\subsection{Inverted structures and quantum wells}

Considerable progress has been made recently in improving the properties of 2DEGs and 2DHGs formed at the inverted interface by reducing the tendency of dopants to diffuse and segregate [11], using low temperature growth techniques [12]. It is now possible to grow a variety of coupled electron-electron and electron-hole gas structures using similar methods. 


\section{Low threshold current lasers}

\subsection{Non radiative recombination centres in (AlGa)As}

Low threshold current lasers are only obtained at very high growth temperatures, using $\mathrm{As}_{4}$, where the re-evaporation rate of gallium is very high [13, 14], but for $\mathrm{As}_{2}$ [15] this is not required. The origin of this difference is not altogether clear, but it has long been established that the growth mechanisms for the $\mathrm{As}_{2}$ [16] and $\mathrm{As}_{4}$ [17] are different. It is also known that at some growth temperatures [18] thick films of ( $\mathrm{AlGa}$ )As prepared using $\mathrm{As}_{4}$ show poor surface morphology, but with $\mathrm{As}_{2}$ this is not observed.

From SIMS studies of thick films of (AlGa)As, we see a clear inverse correlation between the incorporation of oxygen and the photoluminescence (PL) efficiency of the films [19].

To assess the minority carrier properties of our (AlGa)As material, we grow a 60 period intentionally doped multi-quantum-well (MQW) structure. The minority carrier lifetime is measured using a pulsed laser technique and for low threshold current lasers a $10 \mathrm{~ns}$ lifetime for the test structure is essential.

\subsection{Low threshold current lasers}

In our studies using $\mathrm{As}_{2}$, several different types of laser structure were tested, as illustrated in Fig. 2. As shown in Fig. 3, what is most obvious from our data is that the introduction of superlattices (SL) has a clearly beneficial effect and this
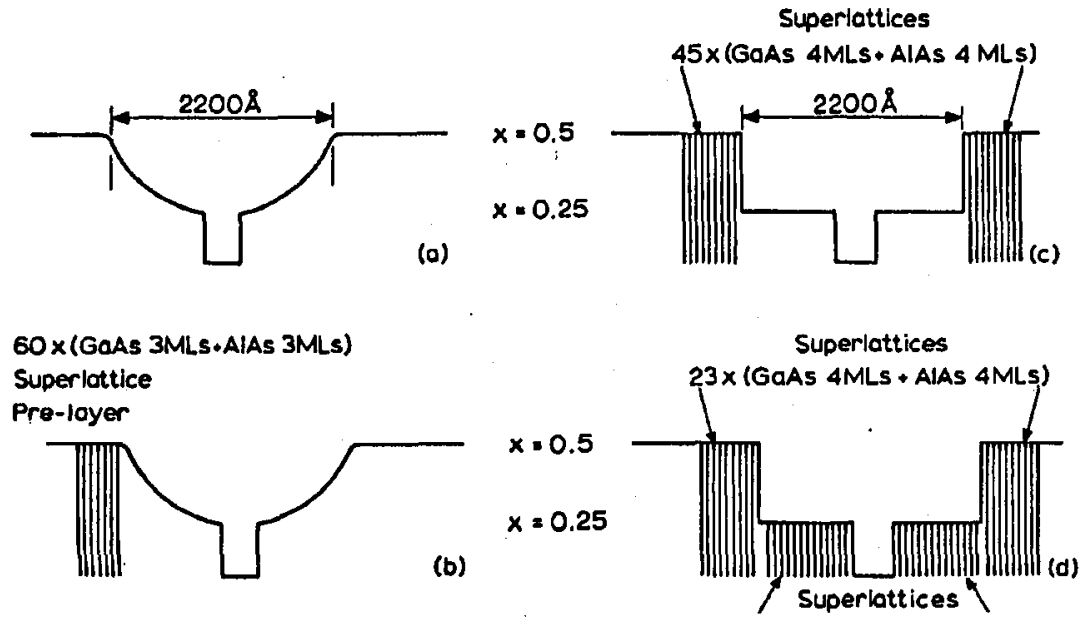

Fig. 2. The variation in the conduction band gap for a variety of graded refractive index and separate confinement heterostructure lasers grown to study the effects of replacing bulk layers with pre-layers or superlattices. All structures were grown using $\mathrm{As}_{2}$. 


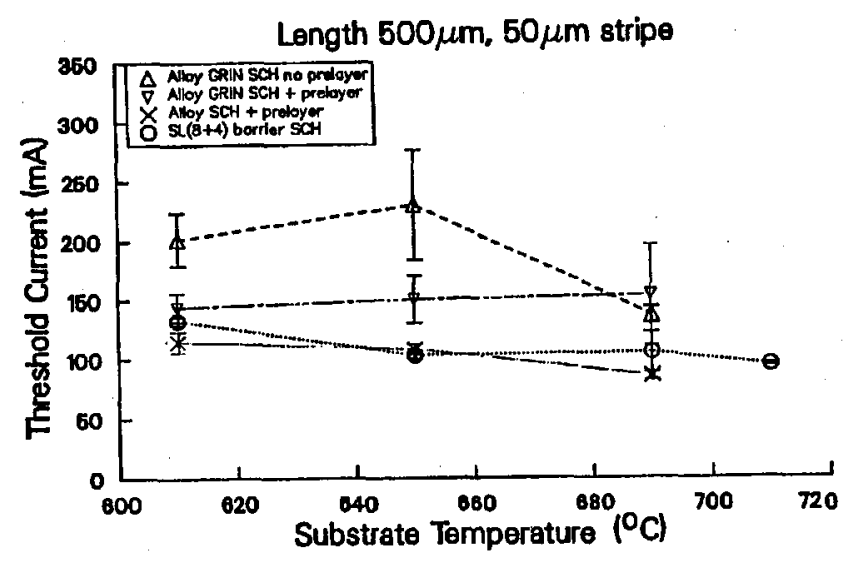

Fig. 3. The variation in threshold current with growth temperature for the various laser structures shown in Fig. 2. The advantages offered by introducing superlattices before the active part of the structure can clearly be seen. In particular little dependence on growth temperature can be observed in contrast to the similar structure grown using $\mathrm{As}_{4}$.

has now been shown to be due to trapping of oxygen at the "inverted interface" [20]. The exact mechanism for this is still not clear, but the observations suggest that, whilst the sticking probability of oxygen on (AlGa)As surfaces is much higher than on GaAs, the oxygen is only slowly incorporated into (AlGa)As films and is efficiently trapped close to the interface in GaAs films grown on top of the (AlGa)As layers.

\section{Applications of MBE to advanced solar cell devices}

In work carried out in collaboration with the group of Dr Barnham at Imperial College, London, we have been studying the growth of (AlGa)As MQW structures for improved solar cell performance. Barnham et al. [21] suggested that an improved performance for solar cells could be obtained by incorporating an undoped MQW region into a $p-i-n$ structure. In principle, if the additional carriers created in the narrow band gap materials in the wells can be extracted efficiently by the field to the wide band gap barrier regions, an enhanced current can be obtained and at the same time the open circuit voltage, characteristic of the wide band gap material can be retained. The possibility of improved performance has been disputed by some authors using a detailed balance theory [22], but is supported by considerations of the radiative efficiency of the cells [23]. Evidence is accumulating that, for practical materials, some gain in overall efficiency is possible using this method.

In the course of this study we discovered that our MBE single QW $p$-i-n structures were significantly inferior to equivalent structures grown by metal-organic vapour phase epitaxy (MOVPE), but MQW structures samples with similar minority carrier lifetimes were obtained using both methods. The MBE grown 
SQW structures were greatly improved by introducing an SL prelayer which might indicate that oxygen(?) or some other impurity able to segregate on the (AlGa)As surface is responsible.

In summary for high quality (AlGa)As/GaAs devices structures, eliminating oxygen from the layers is essential. This limits the performance of all opto-electronic devices especially those dependent upon minority carrier properties such as solar cells.

\section{Conclusions}

To produce high quality low dimensional structures or practical devices by MBE requires attention to detail in the selection of materials used for evaporation and in the correct preparation of the equipment. The complicating factor is that the partial pressure of unwanted impurities is below the detections limit of simple residual gas analyzers used in MBE which remains, therefore, a process needing a skilled operation.

\section{References}

[1] C.T. Foxon, J.J. Harris, Philips J. Res. 41, 313 (1986).

[2] G. Weimann, W. Schlapp, Appl. Phys. Lett. 46, 411 (1985).

[3] G.W.'t Hooft, W.A.J.A. van der Poel, L.W. Molenkamp, C.T. Foxon, Phys. Rev. B 35, 8281 (1987).

[4] C.R. Stanley, M.C. Holland, A.H. Kean, J.M. Chamberlain, R.T. Grimes, M.B. Stanaway, J. Cryst. Growth 111, 14 (1991).

[5] C.T. Foxon, J.J. Harris, D. Hilton, J. Hewett, C. Roberts, Semicond. Sci. Technol. 4, 582 (1989).

[6] L. Pfeiffer, K.W. West, H.L. Stromer, K.W. Baldwin, Appl. Phys. Lett. 55, 1350 (1989).

[7] T. Saku, Y. Hirayama, Y. Horikoshi, Jpn. J. Appl. Phys. 30, 902 (1991).

[8] A.G. Davies, R. Newbury, M. Pepper, J.E.F. Frost, D.A. Ritchie, G.A.C. Jones, Phys. Rev. B 44, 13128 (1991).

[9] M. Henini, P.J. Rodger, P.A. Crump, B.L. Gallagher, G. Hill, Appl. Phys. Lett. 65, 2054 (1994).

[10] W.I. Wang, E.E. Mendez, T.S. Kuan, L. Esaki, Appl. Phys. Lett. 47, 826 (1985).

[11] J.J. Harris, J.B. Clegg, R.B. Beall, J. Castasgne, K. Woodbridge, C. Roberts, J. Cryst. Growth 111, 239 (1991).

[12] T. Sajoto, M. Santos, J.J. Heremens, M. Shayegan, M. Heiblum, M.V. Weckwerth, U. Meirav, Appl. Phys. Lett. 54, 840 (1989).

[13] W.T. Tsang, F.K. Reinhart, J.A. Ditzenberger, Appl. Phys. Lett. 36, 118 (1980).

[14] S.V. Iyer, H.P. Meier, S. Ovadia, C. Parks, D.J. Arent, W. Walter, Appl. Phys. Lett. 60, 416 (1992).

[15] C.T. Foxon, P. Blood, E.D. Fletcher, D. Hilton, P.J. Hulyer, M. Vening, J. Cryst. Growth 111, 1047 (1991).

[16] C.T. Foxon, B.A. Joyce, Surf. Sci. 64, 293 (1977).

[17] C.T. Foxon, B.A. Joyce, Surf. Sci. 50, 434 (1975). 
[18] H. Morkoc, T.J. Drummond, W. Kopp, R. Fischer, J. Electrochem. Soc. 129, 824 (1982).

[19] C.T. Foxon, J.B. Clegg, K. Woodbridge, D. Hilton, P. Dawson, P. Blood, J. Vac. Sci. Technol. B 3, 703 (1985).

[20] T. Achtnich, G. Burri, M.A. Py, M. Ilegems, Appl. Phys. Lett. 50, 1730 (1987).

[21] K.W.J. Barnham, G. Duggan, J. Appl. Phys. 67, 1359 (1990).

[22] G.L. Araujo, A. Marti, in: Proc. 11th. EC Solar Energy Conf., Harwood Academic Publ., 1992, p. 142.

[23] R. Corkish, M.A. Green, in: Proc. 23rd. IEEE Spec PV Conf., IEEE, 1993, p. 675. 